\title{
Evaluation and Improvement of an Iterative Scattering Correction Scheme for in situ Absorption and Attenuation Measurements
}

\author{
DAVID MCKeE \\ Physics Department, University of Strathclyde, Glasgow, United Kingdom \\ JACEK PISKOZUB \\ Institute of Oceanology, Polish Academy of Sciences, Sopot, Poland \\ RÜDIGER RÖTTGERS \\ Helmholtz-Zentrum Geesthacht Centre for Materials and Coastal Research, Geesthacht, Germany \\ RICK A. REYNOLDS \\ Scripps Institution of Oceanography, University of California, San Diego, La Jolla, California
}

(Manuscript received 24 July 2012, in final form 14 December 2012)

\begin{abstract}
The performance of several scattering correction schemes for reflecting-tube absorption and beam attenuation measurements is evaluated with data collected in European shelf seas. Standard scattering correction procedures for absorption measurements perform poorly because of nonzero absorption in the near infrared and wavelength-dependent scattering phase functions. A previously described iterative correction procedure based on Monte Carlo simulations of the Western Environmental Technologies Laboratories (WET Labs) ac-9 and independent estimates of particle backscattering initially performs poorly, but is greatly improved when realistic losses at flow-tube walls are incorporated into the model. The updated Monte Carlo scattering correction provides excellent agreement with independent absorption and attenuation measurements made with a point-source integrating-cavity absorption meter (PSICAM) and a Laser In Situ Scattering and Transmissometer (LISST, Sequoia Scientific), respectively. Implications for historic datasets and requirements for application to future datasets are discussed.
\end{abstract}

\section{Introduction}

The propagation of light through seawater is regulated by the effect of the optical properties of the water itself and of materials suspended and dissolved within the medium. The spectral absorption $a(\lambda)$ and attenuation $c(\lambda)$ coefficients, where $\lambda$ refers to light wavelength in vacuo, are fundamental optical characteristics of the medium that are determined only by these materials themselves and not by the structure of the light field. As such they are often referred to as inherent optical properties (IOPs) of the medium (Mobley 1994).

Corresponding author address: David McKee, Physics Department, University of Strathclyde, 107 Rottenrow, Glasgow G4 0NG, United Kingdom.

E-mail: david.mckee@strath.ac.uk
Measuring the absorption of dissolved substances is normally straightforward and can be easily achieved for normal practical error expectation levels using simple spectrophotometric methods. However, measuring the absorption of turbid media is considerably more complicated because of the effect of scattering on measured signals (Stramski and Piskozub 2003). Various approaches to resolve this issue have been attempted, with the reflecting-tube absorption meter [Western Environmental Technologies Laboratories (WET Labs) ac-9/ ac-spectra (ac-s)] becoming a widely adopted instrument within the optical oceanography community for measuring in situ absorption. This method uses total internal reflectance at the flow-tube walls to redirect a portion of the scattered light toward a diffuser in front of a large-area receiver with the aim of minimizing scattering losses. Even with this setup, it is necessary to 
perform a scattering correction to account for residual scattering losses (Zaneveld et al. 1994). These residual scattering losses can be quite substantial in turbid waters and often dominate the measured signal in some spectral bands with low natural particulate absorption. To date there has not been a thorough validation of the efficacy of the various proposed scattering correction methods for WET Labs ac-9 absorption data using field data.

The attenuation of seawater is measured by focusing a transmitted collimated beam of light onto a small aperture and detecting the photons that have not been absorbed or scattered, $b(\lambda)$, with $c(\lambda)=a(\lambda)+b(\lambda)$. As a result of the aperture having a finite diameter, the optical arrangement has a characteristic collection angle $\psi_{c}$ that is a source of scattering error for the attenuation measurement. Piskozub et al. (2004) have shown that the scattering error for this type of transmissometer is a simple function of the number of photons scattered between 0 and $\psi_{c}$, even for rather turbid waters. There have been few attempts to correct beam attenuation measurements for the forward-scattering collection error (Voss and Austin 1993). Boss et al. (2009) recently demonstrated that uncorrected WET Labs ac-9 attenuation values are approximately $50 \%-80 \%$ of equivalent Laser In Situ Scattering and Transmissometer (LISST) attenuation data, with the two instruments having inwater scattering collection angles of $\sim 0.9^{\circ}$ and $\sim 0.027^{\circ}$ respectively.

Several studies have examined various aspects of the absorption and attenuation meter designs including optimization of flow-tube diameter and reflecting materials for the absorption tube, effects of multiple scattering, and potential errors in attenuation measurements through incomplete exclusion of forward scattering (Kirk 1992; Hakvoort and Wouts 1994; Piskozub et al. 2001, 2004). Field studies have noted potential limitations in the quality of ac- 9 absorption data using optical closure with in situ radiometry as a test (McKee et al. 2003; McKee and Cunningham 2005). There is a growing realization of the need to understand and quantify uncertainties associated with these measurements across the wide range of optical conditions in which the instruments are deployed (Doxaran et al. 2007; Leymarie et al. 2010).

McKee et al. (2008) presented an iterative scattering correction scheme for both in situ absorption and attenuation measurements based on Monte Carlo simulations of the WET Labs ac-9 optical layout. The Monte Carlo (MC)-based approach requires concurrent measurements of particulate backscattering, $b_{\mathrm{bp}}(\lambda)$ to facilitate estimation of the scattering phase function as part of the iterative correction process. At the time of publication, it was impossible to assess the performance of the proposed method, as there was no means of making an independent, validated absorption measurement. Since then a new dataset has been collected that permits comparison of ac-9 data with data from a point source integrating cavity absorption meter (PSICAM), developed at Helmholtz-Zentrum Geesthacht (HZG). This instrument has been rigorously tested and demonstrated to provide accurate absorption measurements over a wide range of optical conditions, including highly turbid waters (Röttgers et al. 2005, 2007; Röttgers and Doerffer 2007). At the same time a small set of concurrent ac-9 and LISST attenuation data have been collected. The LISST has a much smaller collection angle than the ac- 9 transmissometer and therefore ought to be subject to a considerably smaller scattering collection angle error. However, since this error would still be nonzero, we have used the same approach to correct the LISST data, enabling us to test the consistency of ac-9 and LISST attenuation values after both have been corrected in the same manner.

The purpose of this paper is first of all to assess the performance of the MC scattering correction scheme by comparing in situ ac-9 absorption and attenuation data with concurrent PSICAM absorption and LISST attenuation data. Data are presented from three European shelf seas and from two different sets of in situ IOP sensors, that is, two ac- 9 instruments and two backscattering meters. In doing so, it is possible to establish the general applicability of the MC-based approach and its performance over a range of conditions that is of interest to a broad section of the oceanography community.

\section{Methods}

\section{a. Optical measurements}

Absorption and attenuation coefficients were measured with two $25-\mathrm{cm}$-pathlength ac-9 instruments with slightly different wavelength sets. The University of Strathclyde (UoS) ac-9 was equipped with filters at 412 , $440,488,510,532,555,650,676$, and $715 \mathrm{~nm}$, while the HZG ac-9 replaced $532 \mathrm{~nm}$ with $630 \mathrm{~nm}$. Both ac-9 units were calibrated with ultrapure water (Milli-Q, Millipore) and samples were corrected for temperature $(T)$ and salinity $(S)$ dependence of pure seawater (Pegau et al. 1997). A WET Labs BB9 backscattering meter $(412,440,488,510,532,595,650,676$, and $715 \mathrm{~nm})$ was deployed with the UoS ac-9, while a Hydro-Optics, Biology \& Instrumentation Laboratories (HOBI Labs) Hydroscat-6 (HS-6; 442, 470, 550, 589, 620, and $671 \mathrm{~nm}$ ) and two single-wavelength a- $\beta$ eta backscattering meters (420 and $510 \mathrm{~nm}$ ) were deployed with the HZG ac-9. Backscattering data were interpolated to match ac-9 
wavelengths when necessary. Absorption and scattering coefficients for pure water were taken from Pope and Fry (1997) and Smith and Baker (1981), respectively. The HOBI Labs HS- 6 and a- $\beta$ eta measure the spectral volume scattering function (VSF), $\beta(\psi, \lambda)$, at an effective scattering angle of $\psi=140^{\circ}$ (Maffione and Dana 1997). The backscattering coefficient was determined from the measured $\beta\left(140^{\circ}, \lambda\right)$ using the procedures described in detail by Stramski et al. (2008). Theoretical estimates of pure seawater scattering, adjusted for temperature and salinity effects, were calculated from the relationships described in Buiteveld et al. (1994) and Twardowski et al. (2007). The estimated $b_{b}(\lambda)$ in each spectral band was corrected for signal attenuation along the pathlength utilizing independent estimates of absorption from the PSICAM and scattering from the ac-9. The BB9 measures the spectral volume scattering function $\beta(\psi, \lambda)$ at an effective scattering angle of $\psi=117^{\circ}$. The backscattering coefficient was determined from the measured $\beta\left(117^{\circ}, \lambda\right)$ using the procedures described in detail in the BB9 manual. These can be briefly summarized as including the following steps: (i) correction for pathlength absorption using ac- 9 absorption data corrected using the Zaneveld method (Zaneveld et al. 1994), (ii) conversion of total VSF to particulate VSF by subtraction of water VSF determined from Morel (1974), and (iii) conversion to backscattering coefficient from $\beta\left(117^{\circ}, \lambda\right)$ using the relationship determined by Boss and Pegau (2001) and a $\chi$ factor of 1.1 .

Uncorrected ac-9 absorption and attenuation signals are subject to systematic errors associated with incomplete collection and rejection of scattered photons, respectively. Because of the fundamental limitations of all transmissometer designs, the manufacturer recommends no scattering correction method for attenuation measurements and a number of possible scattering correction methods for absorption data. We note that these cannot all be correct simultaneously, as they inevitably provide different values for a given sample. The first suggestion is to make a "Flat" baseline correction by subtracting the measured signal (after $T$ and $S$ correction) at a reference wavelength, $a_{m}\left(\lambda_{r}\right)$, where the absorption by dissolved and particulate material is assumed to be zero (usually $715 \mathrm{~nm}$ ):

$$
a_{n}(\lambda)=a_{m}(\lambda)-a_{m}\left(\lambda_{r}\right),
$$

where $a_{m}(\lambda)$ is the signal measured at each wavelength $\lambda$ following water subtraction and $T$ and $S$ correction. A commonly used alternative suggestion from the ac- 9 manual is the proportional correction method devised by Zaneveld et al. (1994) (hereafter "Zaneveld"), which weights the measured signal at the reference wavelength by the ratio of measured scattering at the signal and reference wavelengths:

$$
a_{n}(\lambda)=a_{m}(\lambda)-a_{m}\left(\lambda_{r}\right) \frac{c_{m}(\lambda)-a_{m}(\lambda)}{c_{m}\left(\lambda_{r}\right)-a_{m}\left(\lambda_{r}\right)},
$$

where $a_{n}(\lambda)$ is the scattering-corrected, nonwater absorption; $a_{m}(\lambda)$ is the measured absorption signal; $c_{m}(\lambda)$ is the measured attenuation signal after both $T$ and $S$ correction; and $\lambda$ and $\lambda_{r}$ are the signal and reference wavelengths, respectively.

An iterative scattering correction procedure for ac-9 absorption and attenuation data based on 3D Monte Carlo simulations of the ac-9 optical configuration was recently presented (McKee et al. 2008) and is summarized here. The absorption sensor fails to collect all scattered light and the attenuation sensor fails to exclude all scattered photons, so that true nonwater absorption $a_{n}(\lambda)$ and attenuation $c_{n}(\lambda)$ signals are given by

$$
\begin{aligned}
& a_{n}(\lambda)=a_{m}(\lambda)-f_{a}(\lambda) b_{p}(\lambda) \text { and } \\
& c_{n}(\lambda)=c_{m}(\lambda)+f_{c}(\lambda) b_{p}(\lambda),
\end{aligned}
$$

where $b_{p}(\lambda)$ is the true particulate scattering coefficient, $f_{a}(\lambda)$ is the fraction of scattered light not collected by the absorption sensor, and $f_{c}(\lambda)$ is the fraction of scattered light that is collected by the attenuation sensor. As the true particulate scattering is given by $b_{p}(\lambda)=c_{n}(\lambda)-$ $a_{n}(\lambda)$, Eqs. (3) and (4) can be used to demonstrate that

$$
\begin{aligned}
& b_{p}(\lambda)=\frac{b_{m}(\lambda)}{1-f_{a}(\lambda)-f_{c}(\lambda)}, \\
& a_{n}(\lambda)=a_{m}(\lambda)-\frac{f_{a}(\lambda) b_{m}(\lambda)}{\left[1-f_{c}(\lambda)-f_{a}(\lambda)\right]}, \text { and } \\
& c_{n}(\lambda)=c_{m}(\lambda)+\frac{f_{c}(\lambda) b_{m}(\lambda)}{\left[1-f_{c}(\lambda)-f_{a}(\lambda)\right]} .
\end{aligned}
$$

The fraction $f_{a}(\lambda)$ is given by

$$
f_{a}(\lambda)=2 \pi \int_{0}^{\pi} W_{a}(\psi) \tilde{\beta}_{p}(\lambda, \psi) \sin \psi d \psi,
$$

where $\tilde{\beta}_{p}(\lambda, \psi)$ is the wavelength-dependent particulate scattering phase function and $W_{a}(\psi)$ is a wavelengthindependent weighting distribution function that describes the angular scattering collection efficiency of the absorption sensor (Stramski and Piskozub 2003). Piskozub et al. (2004) have shown that the equivalent weighting function for attenuation sensors is reasonably approximated by assuming that all photons scattered 
between zero and the collection angle of the transmissometer $\left(\psi_{c}\right)$ contribute equally to the attenuation scattering error and that photons scattered outside this range make zero contribution to the error. This was confirmed using the same Monte Carlo simulation code for the ac- 9 attenuation sensor configuration $\left(0.9^{\circ}\right.$ halfwidth collection angle in water; not shown). Therefore, $f_{c}(\lambda)$ is given by the cumulative phase function from zero to $\psi_{c}$ :

$$
f_{c}=2 \pi \int_{0}^{\psi_{c}} \tilde{\beta}_{p}(\lambda, \psi) \sin \psi d \psi
$$

To estimate $f_{a}(\lambda)$ and $f_{c}(\lambda)$, the iterative scattering correction procedure requires estimation of $\tilde{\beta}_{p}(\lambda, \psi)$. This is obtained using the Mobley et al. (2002) parameterization for Fournier-Forand scattering phase functions and $b_{\mathrm{bp}}(\lambda) / b_{p}(\lambda)$ derived from in situ measurements of particulate backscattering, $b_{\mathrm{bp}}(\lambda)$ and $b_{p}(\lambda)$. It should be noted, however, that alternative scattering phase function parameterizations could be assimilated into the procedure if necessary (Freda and Piskozub 2007). Moreover, Fournier-Forand phase functions are based on an assumption of a power-law particle size distribution whose validity has been challenged in a number of studies (Jonasz and Fournier 1996; Risović 2002; Reynolds et al. 2010).

Monte Carlo simulations of the ac-9 absorption and attenuation tubes enabled estimation of fractional scattering collection of each sensor as functions of the particulate backscattering ratio. To use this information to correct ac- 9 signals, one has to be able to estimate the particulate backscattering ratio, $b_{\mathrm{bp}}(\lambda) / b_{p}(\lambda)$. However, since this involves use of particulate scattering, $b_{p}(\lambda)$, a potential logical circularity is encountered. This problem was overcome by adopting an iterative scattering error correction approach whose steps are outlined in Fig. 1. These steps include initial estimate of $b_{p}(\lambda)$ using a typical value of 1.5 for $1 /\left[1-f_{a}(\lambda)-f_{c}(\lambda)\right]$, initial estimation of $b_{\mathrm{bp}}(\lambda) / b_{p}(\lambda)$ leading to estimates of $c_{n}(\lambda)$ and $a_{n}(\lambda)$, and iteration until $b_{\mathrm{bp}}(\lambda) / b_{p}(\lambda)$ reaches a stable value within a set tolerance. The choice of tolerance is arbitrary, but it should be sufficiently small to ensure adequate parameterization of $f_{a}(\lambda)$ and $f_{c}(\lambda)$, and large enough to facilitate closure within a reasonable number of iterations. A threshold of 0.001 was chosen for initial testing.

The reference "true" absorption was measured using a PSICAM (Röttgers et al. 2005, 2007; Röttgers and Doerffer 2007). Samples of about $500 \mathrm{~mL}$ were measured against purified water as the reference with a calibrated instrument. Each sample was poured into the PSICAM's cavity and the light intensity inside the cavity

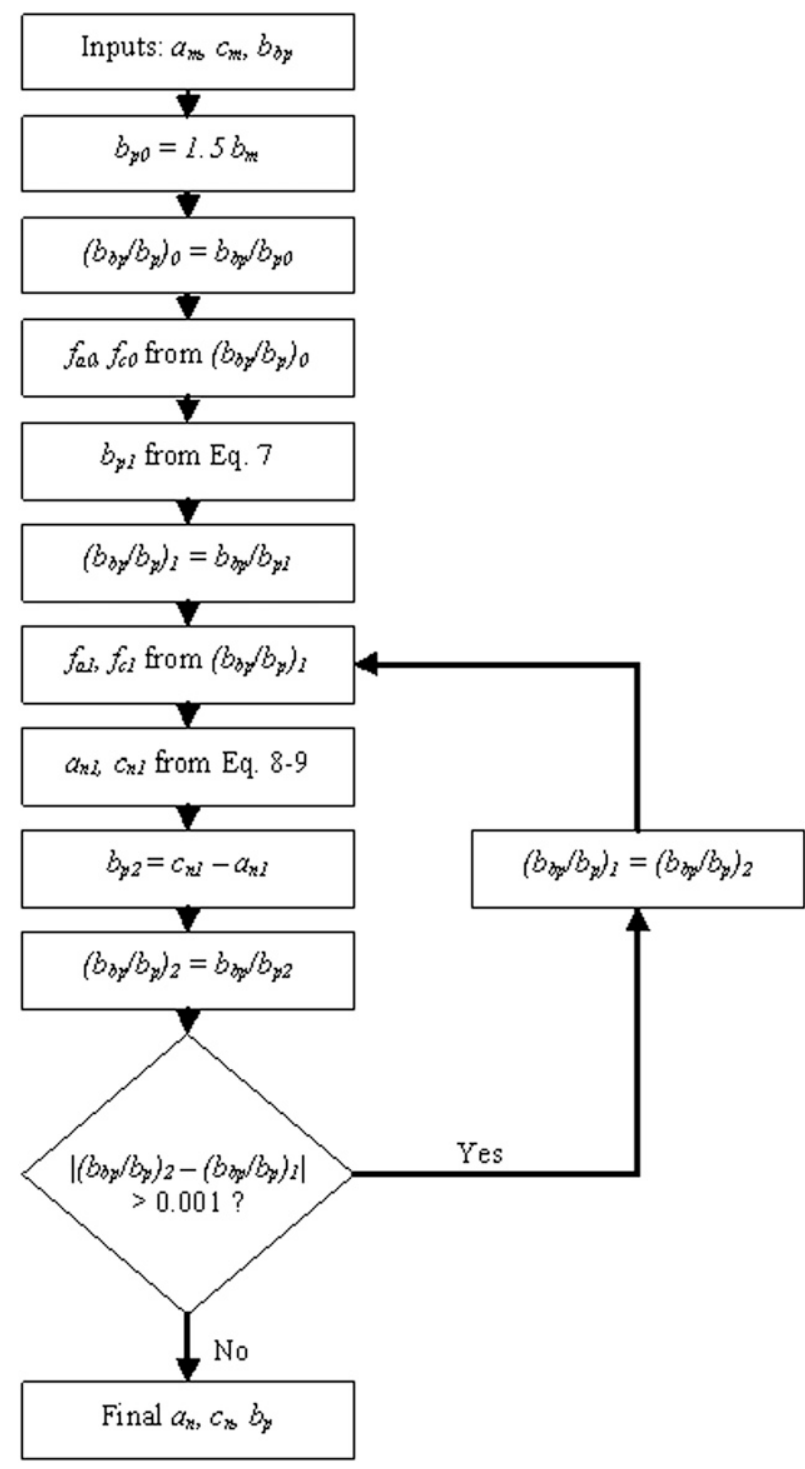

FIG. 1. Schematic diagram of steps for the iterative MC-based scattering correction for ac-9 absorption and attenuation data.

was measured with a spectroradiometer. The reference light intensity was measured before and after the sample when purified water was poured into the cavity. The absorption was calculated from the "transmission" (sample/reference) and corrected for chlorophyll fluorescence, and for temperature and salinity differences between sample and reference using instrument-specific correction coefficients. Calibration of the PSICAM was performed before and after each cruise using dye solutions whose absorption was measured in a spectrophotometer using a 10-cm cuvette. For absorption coefficients between 0.01 and $5 \mathrm{~m}^{-1}$, the precision of the PSICAM was similar to or better than that of the ac-9. The overall error for PSICAM data is determined by the calibration 
and was between $1 \%$ and $2 \%$ when absorption was $>0.01 \mathrm{~m}^{-1}$. The determination with a PSICAM is not sensitive to errors induced by bubbles or other sources of scattering (Röttgers et al. 2005, 2007; Röttgers and Doerffer 2007).

The Scripps Institution of Oceanography (SIO) LISST100X (type B, Sequoia Scientific) measures scattering and attenuation of a 532-nm laser beam as it traverses a $5-\mathrm{cm}$ pathlength. For the data utilized in this study, a sample chamber was inserted into the optical head of the instrument and measurements of beam attenuation were conducted on discrete water samples collected using either a Niskin bottle or a bucket. Blank determinations were made by measuring seawater passed through a $0.22-\mu \mathrm{m}$ filter to remove particles, and subsequently subtracted from the sample measurements. About 500 individual scans were recorded and averaged for each sample, with the variability among scans generally observed to be $<5 \%$. Reynolds et al. (2010) provide further details of the LISST measurement procedures.

Data were collected on three cruises: the first in the Baltic Sea and the North Sea in September 2007 on board the R/V Oceania, the second in the Mediterranean Sea (Ligurian Sea) in March 2009 on board the $\mathrm{R} / \mathrm{V}$ Alliance, and the third in the North Sea in May 2010 on board the R/V Heincke. The HZG ac- 9 and SIO backscattering meters were deployed on the Baltic/ North Sea cruise along with the LISST instrument, while the UoS ac-9 and BB9 were deployed on the Mediterranean Sea cruise. Both the UoS and HZG ac-9 instruments were deployed on the North Sea 2010 cruise. Profiles of salinity and temperature with depth were obtained from SeaBird CTDs on each cruise. Water samples were collected from surface waters at selected depths and their absorption measured on board the ship using the PSICAM. Each cruise sampled a variety of optical water conditions including turbid coastal waters and relatively clear, open waters.

\section{b. Monte Carlo modeling}

Monte Carlo simulations were performed with code previously used to study ocean optics phenomena and instrumentation (Stramski and Piskozub 2003; Piskozub et al. 2004; Flatau et al. 1999; Piskozub et al. 2008). The ac- 9 absorption and attenuation sensors were simulated using accurate instrument dimensions provided by the manufacturer. The pathlength of each sensor $(\tau)$ was $0.25 \mathrm{~m}$ and the transmissometer had a scattering collection half-width angle of $0.9^{\circ}$. The effect of imperfections in the structure of the glass cuvette walls of the ac- 9 absorption tube was modeled by randomly imposing a percentage error loss term $r_{w}$ on photons striking the water-glass interface from both directions. Simulations were performed for $10^{8}$ photons per run and the following IOPs: $a=0.006 \mathrm{~m}^{-1}, b=0.25 \mathrm{~m}^{-1}$, and a FournierForand phase function calculated using the Mobley et al. (2002) parameterization with $b_{\mathrm{bp}} / b_{p}=0.02$. It should also be noted that IOPs were selected to ensure that the optical thickness $(c \tau)$ was less than 0.3 , so that multiple scattering could be ignored (van de Hulst 1957) and sufficient numbers of photons would be scattered into each angular bin to facilitate statistical analysis. By definition, the $W_{a}$ function is independent of the IOPs used in the Monte Carlo simulations and can be used with other combinations of IOPs and phase functions without further modification, with the possible exception of strongly multiple scattering waters (Stramski and Piskozub 2003). This was confirmed by a series of simulations with varying absorption, scattering, and scattering phase functions that were performed for a given ac-9 absorption configuration (Fig. 2). Piskozub and McKee (2011) have recently demonstrated a simple method of determining the effective scattering phase function for the multiple scattering regime, which potentially could be used to extend the range of this correction method into more turbid waters.

\section{Results}

The performance of the original MC-based iterative scattering correction for ac-9 absorption data was tested by comparing with PSICAM absorption from samples collected on each of the cruises (Fig. 3). In all cases the MC-corrected ac-9 data generally overestimated the true (PSICAM) absorption at all wavelengths. Average errors for each cruise varied between 0.05 and $0.1 \mathrm{~m}^{-1}$ (RMSE), but the maximum errors were greater than an order of magnitude (32 fold). Data spread varies between cruises, but this could not be simply attributed to calibration differences between ac- 9 sensors, as both ac-9 instruments were calibrated to essentially equivalent tolerances using ultrapure water from the same Milli-Q system. On the Baltic/North Sea cruise ac-9 and PSICAM measurements were performed on the same discrete water samples. However, on the Mediterranean and North Sea 2010 cruises, ac-9 measurements were made in situ, which may explain to some extent the increase in variability with PSICAM data between cruises.

The observed general overestimation of absorption and its variability with instrument and/or location forced a reassessment of the fundamental assumptions underpinning the MC scattering correction approach. The potential impact of multiple scattering on retrieved absorption signals was considered but quickly eliminated using Monte Carlo simulations as a viable source of the type of errors seen in Fig. 3. Close examination of the 

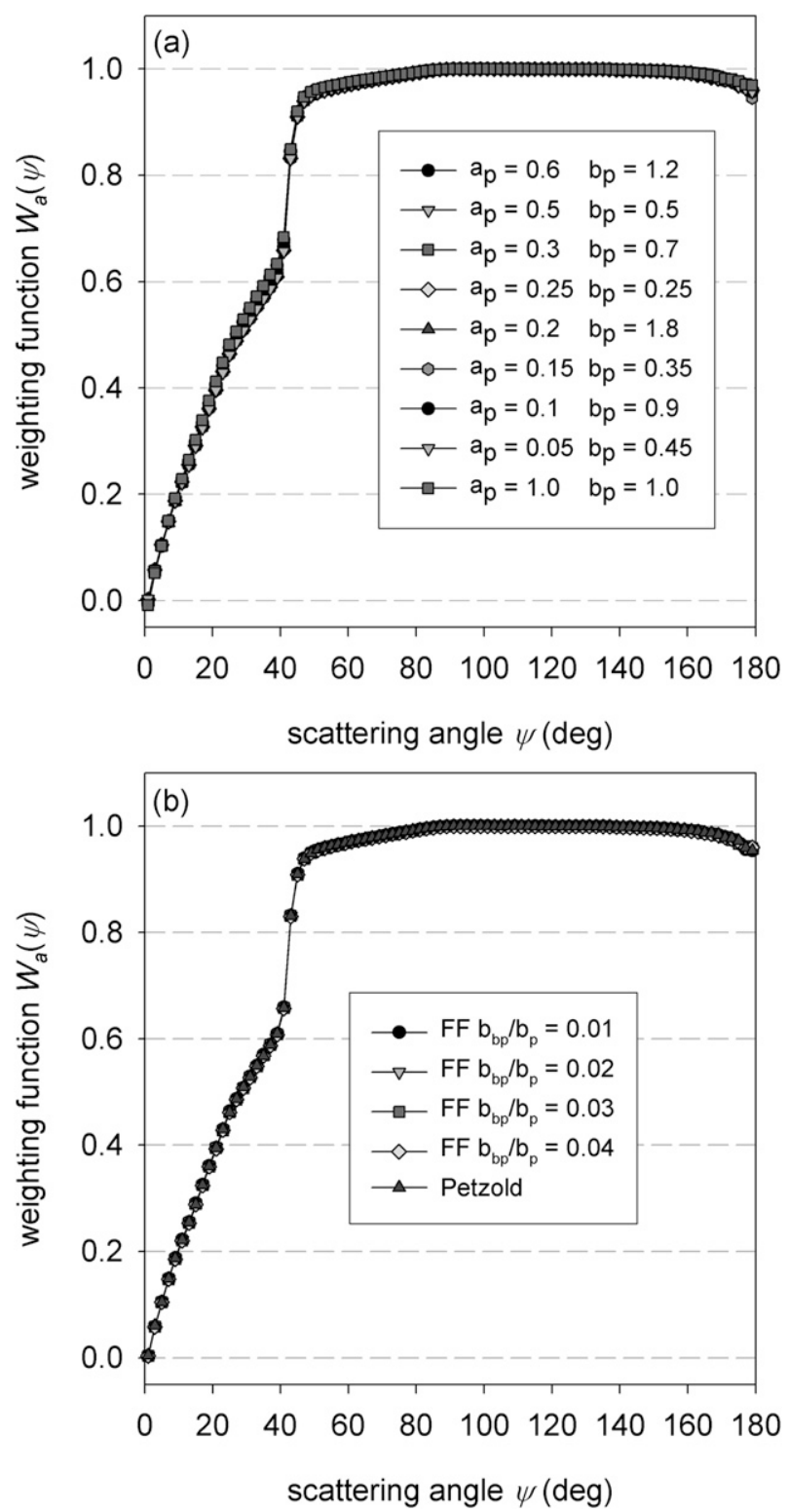

FIG. 2. Monte Carlo simulations for the ac- 9 absorption tube with wall reflectance $r_{w}=0.95$ were repeated for a series of combinations of (a) absorption and scattering coefficients, and (b) scattering phase functions. These results clearly demonstrate the independence of $W_{a}(\psi)$ from the IOPs used in simulations.

ac-9 flow tubes raised the question of the potential impact of imperfections on the glass tube walls on the scattering collection efficiency of the reflecting flow tube. It was also noted that the system relies on total internal reflection occurring at the external surface of the glass cuvette. Therefore, there may be a loss associated with the cuvette being mechanically held in place with O-rings inside the black casing of the flow tube. There is an air gap surrounding the quartz tube that provides the refractive index difference leading to a critical angle of $41.7^{\circ}$ for total internal reflection. Since the exterior surface of the glass flow tube cannot be examined or maintained by the user, there is potential for degradation in reflectance with time.

To test the hypothesis of flow tube "aging," a set of experiments was carried out using three different glass flow tubes with the same ac- 9 sensor. The ac-9 was calibrated with ultrapure water with each of the glass flow tubes in turn. Samples from the North Sea were repeatedly measured with each of the glass flow tubes to obtain mean uncorrected absorption spectra for each sample/flow-tube combination. Figure 4 shows a typical set of results. Highest absorption values were consistently obtained with the oldest, most heavily used glass flow tube; intermediate values were obtained with an old but virtually unused flow tube; and lowest values were obtained with a brand-new glass flow tube. High values of uncorrected absorption correspond with lower values of flow-tube reflectance, so these results support the idea of flow-tube reflectance deterioration with time and use.

Monte Carlo simulations of the reflective tube absorption meter were repeated with an additional loss term representing an effective percentage reflectance efficiency $r_{w}$ for photons that would otherwise have experienced reflectance at the flow-tube wall. Figure 5 shows scattering weighting functions $W_{a}(\psi)$ for the reflecting-tube absorption meter for effective reflectance efficiencies ranging between $100 \%\left(r_{w}=1\right)$ and $95 \%$ $\left(r_{w}=0.95\right)$. Reducing the glass flow-tube wall reflectance efficiency has a profound impact on $W_{a}(\psi)$ for forward-scattering angles from $0^{\circ}$ to $41.7^{\circ}$, the angle for total internal reflectance; $W_{a}(\psi)$ for $r_{w}=1$ is the original function used previously for the result in Fig. 3. As $r_{w}$ is reduced, fewer forward-scattered photons are collected by the absorption meter and the shape of $W_{a}(\psi)$ with scattering angle changes significantly in the forward direction. This distribution can be accounted for by noting the effect of total internal reflection being restricted to angles less than $41.7^{\circ}$ and the reducing influence of wall reflectance for light scattered at near-forward angles. To incorporate this sensitivity to imperfect wall reflectance into the MC scattering correction, each of these $W_{a}(\psi)$ functions was processed with Eq. (8) together with Fournier-Forand scattering phase functions calculated using the Mobley et al. (2002) parameterization for values of $b_{\mathrm{bp}} / b_{p}$ between 0.001 and 0.1. Figure 6 shows the impact of imperfect wall reflectance on the fraction of scattered light $f_{a}$ not collected by the ac- 9 absorption meter as a function of $b_{\mathrm{bp}} / b_{p}$. A best-fit sixth-order polynomial was obtained for each set of $f_{a}$-versus- $b_{\mathrm{bp}} / b_{p}$ data with values given in Table 1 . The range of $b_{\mathrm{bp}} / b_{p}$ shown in Fig. 6 covers the vast majority of values 

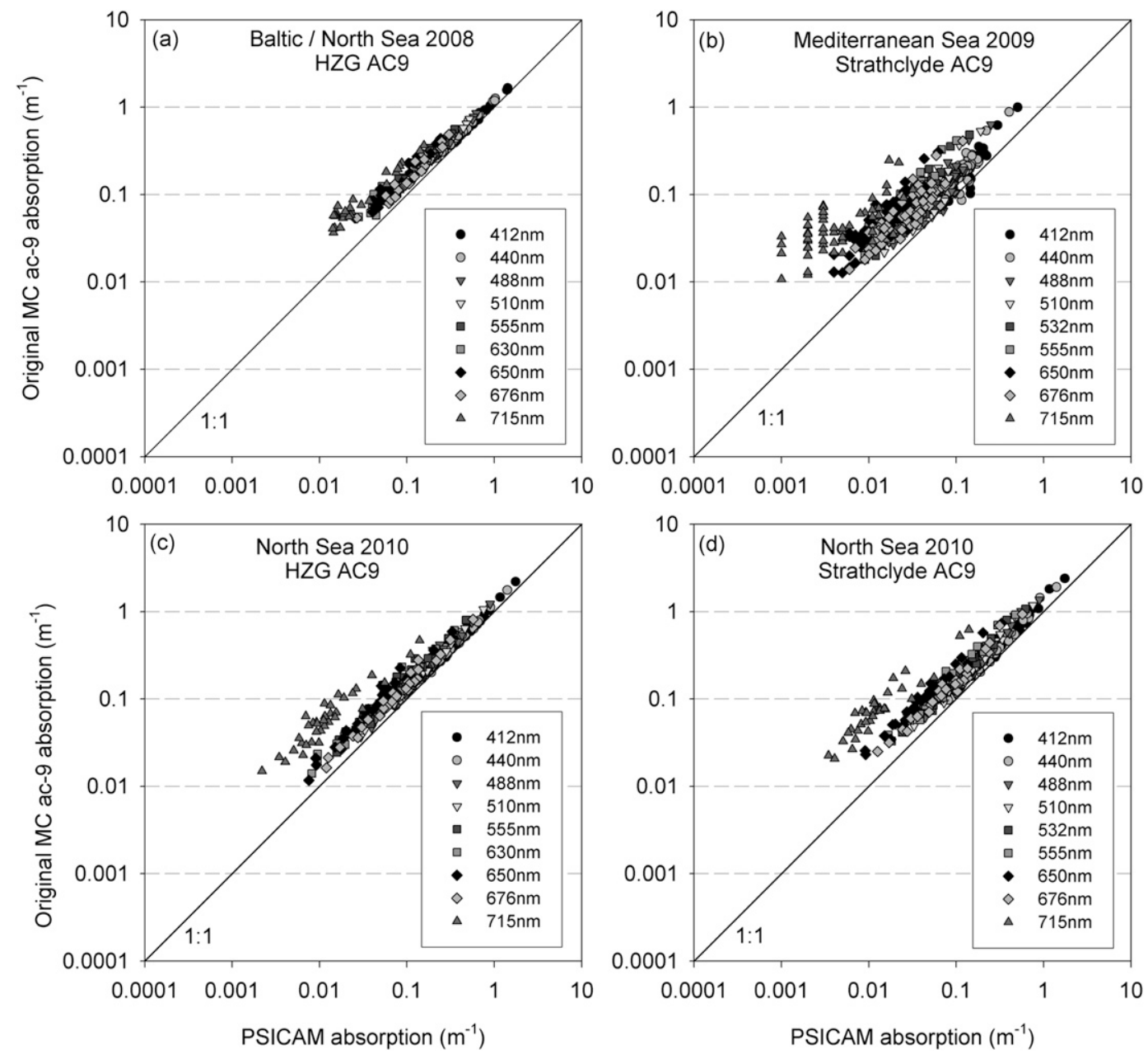

FIG. 3. Absorption coefficients from the ac-9 corrected using the original MC-based scattering correction $\left(r_{w}=1\right)$ compared with PSICAM absorption coefficients for three cruises in European shelf seas. The original MC scattering correction overestimates absorption at all wavelengths.

encountered in natural waters. Ranges of $b_{\mathrm{bp}} / b_{p}$ for the three datasets presented here were $0.002-0.03$.

Figure 6 also shows the fraction of scattered light collected by the attenuation meter, $f_{c}$, as a function of $b_{\mathrm{bp}} / b_{p}$, reproduced from the original analysis (McKee et al. 2008). This remains unchanged and the best-fit function is given by

$$
f_{c}=\frac{6.809 \times 10^{-3}}{8.502 \times 10^{-3}+b_{\mathrm{bp}} / b_{p}}-1.918 \times 10^{-2} .
$$

An additional step now has to be introduced into the MC scattering correction method: determination of $r_{w}$. This is easily achieved where there is access to independent validation data, in this case from the PSICAM. Optimal values of $r_{w}$ were obtained for each cruise dataset by iterating through each of the $f_{a}$ versus $b_{\mathrm{bp}} / b_{p}$ relationships shown in Fig. 6, implementing the $\mathrm{MC}$ scattering correction and selecting the dataset with minimal divergence from the corresponding PSICAM data. Figure 7 shows new estimates of ac- 9 absorption from the MC scattering correction plotted against PSICAM absorption for each of the cruises. In all cases, the systematic overestimation of absorption observed with the original MC scattering correction has been largely eliminated. Average errors for each cruise have been reduced to $<0.025 \mathrm{~m}^{-1}$ (RMSE), though orders of magnitude errors are still observed for individual data points when signal levels are very low $\left(<\sim 0.02 \mathrm{~m}^{-1}\right)$. The relatively strong impact of apparently small changes in wall reflectance can be explained by noting that the greatest influence on $W_{a}$ is at angles between $0^{\circ}$ and $41.7^{\circ}$, where the scattering phase function shape increases very rapidly toward forward angles. 


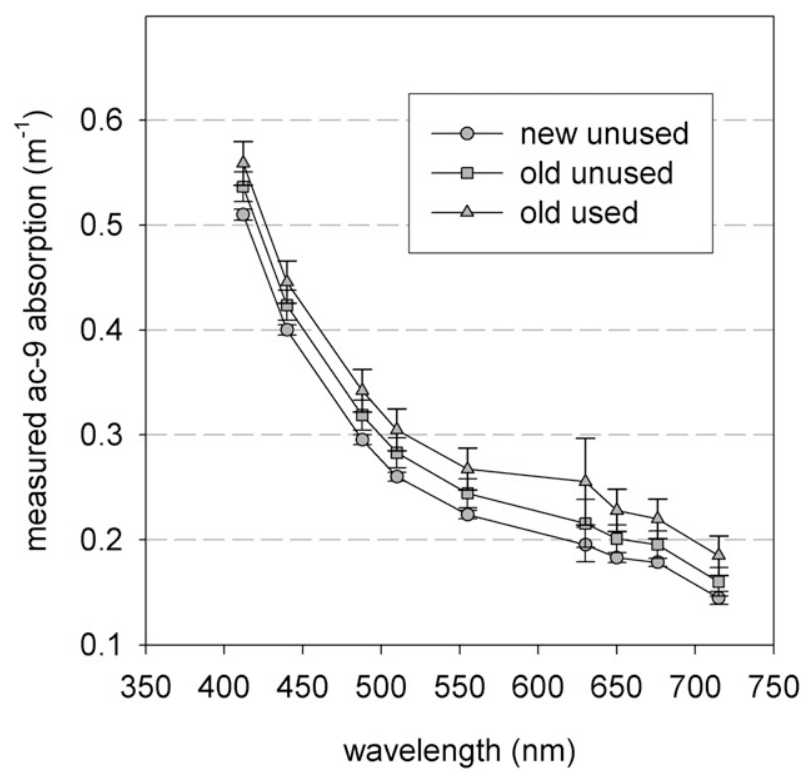

FIG. 4. Uncorrected ac-9 absorption values for a single sample from the North Sea measured with three different glass flow tubes and a single ac- 9 unit. Highest absorption values, corresponding to the lowest wall reflectance values, were obtained with the oldest, most heavily used flow tube, with newer and less used flow tubes giving lower absorption, higher wall reflectance values. Each series shows the mean and standard deviation from an approximately 30 -s collection period $(N \approx 180)$.

It is important to point out that optimal fits with PSICAM absorption data were obtained by selecting different values of $r_{w}$. The optimal $r_{w}$ value for the UoS ac-9 is consistent over two cruises at 0.960 . The optimal value of $r_{w}$ for the HZG ac-9 changed from 0.985 to 0.970 between cruises. This change is most probably associated with the use of a different set of flow tubes for the HZG ac-9 between these cruises. The flow tubes used on the HZG instrument in the North Sea 2010 were older and had been heavily used previously, which supports the idea of potential deterioration over time/ usage. The UoS ac-9 is an older instrument that was not used between the Mediterranean Sea and North Sea 2010 cruises. Long-term stability of the $r_{w}$ value for this instrument will be monitored in the future. The fact that there is a significant difference in $r_{w}$ required for two ac-9 units used on the North Sea 2010 cruise supports the argument that there may be important differences between individual instruments. It is also worth noting that a single value of $r_{w}$ has been shown to provide an optimal match with PSICAM absorption data at nine wavelengths across the visible-near infrared (NIR) for each ac-9 unit tested. In our view, it is unlikely that such a fit could be achieved, particularly for two independent instruments, if the mechanism used did not reflect some basic feature of the two instruments' performance.

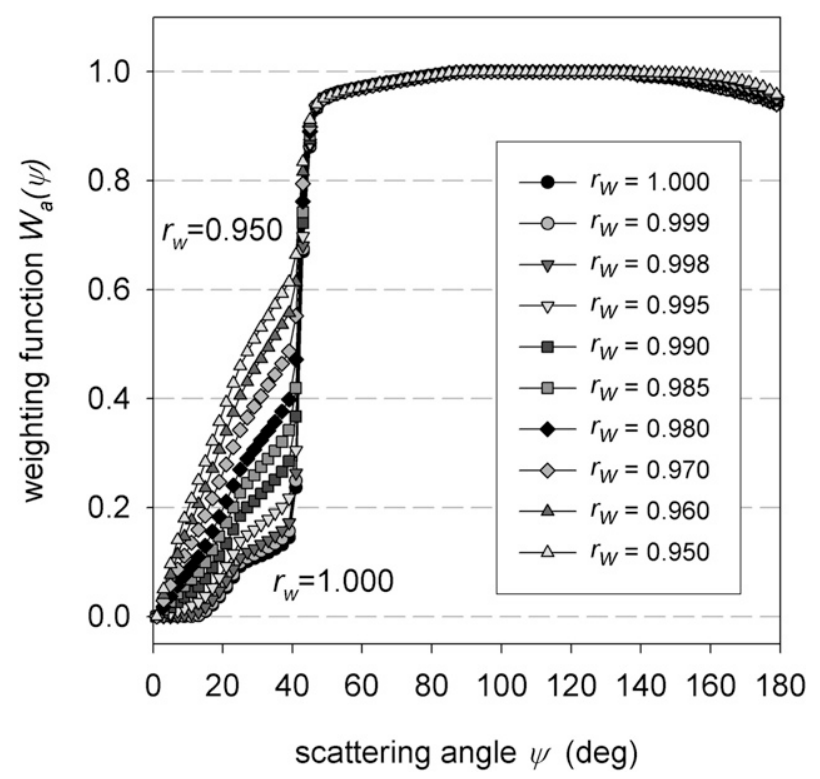

FIG. 5. Scattering weighting function for the ac-9 absorption meter is highly sensitive to reflectance losses (e.g., from imperfections on the flow-tube wall) for forward-scattering angles up to the angle for total internal reflectance $\left(41.7^{\circ}\right) ; r_{w}$ is the modeled fractional reflectance efficiency for the ac- 9 absorption tube wall.

A further check is to examine the spectral quality of the data produced by the optimized Monte Carlo scattering correction. Figure 8 shows one set of absorption spectra taken from each cruise and illustrates the impact of different scattering correction procedures on the measured ac-9 data. Corresponding PSICAM absorption spectra represent best estimates of the true absorption spectrum. Note that there is an order of magnitude difference in the $y$ range between the two datasets. The first point to note is that, in both cases, the uncorrected ac-9 absorption spectrum is significantly greater than the PSICAM spectrum. The scattering correction required to transform measured ac-9 spectra to match PSICAM spectra involves removal of a large proportion of the measured ac- 9 signal. Second, both the Flat and the Zaneveld scattering corrections assume zero nonwater absorption at $715 \mathrm{~nm}$. This does not induce a significant error in fairly clear waters such as the Mediterranean Sea sample (Fig. 8b), it but does result in a significant, systematic underestimate across green-red wavelengths in more turbid waters, such as the Baltic/North Sea sample (Fig. 8a). In both cases, the Flat and Zaneveld corrections give spectral shapes that do not quite match the PSICAM spectrum. The optimized MC scattering correction provides an excellent fit to the PSICAM data across the entire spectrum for the turbid Baltic/North Sea sample (Fig. 8a). The spectral fit is also reasonably good for the clear Mediterranean Sea sample (Fig. 8b), 


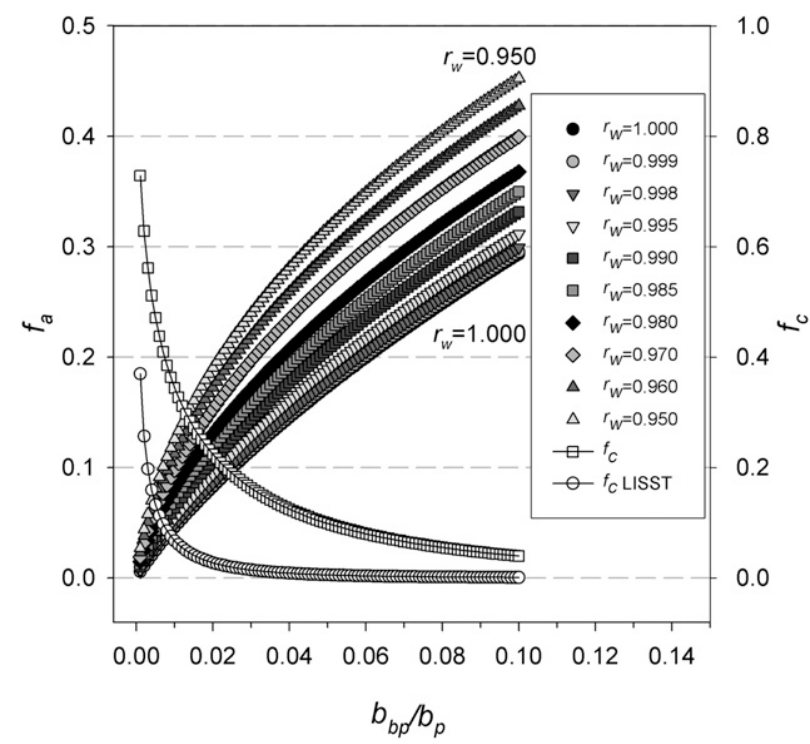

FIG. 6. Fraction of scattered light not collected by the ac-9 absorption meter, $f_{a}$, and collected by the ac- 9 attenuation meter, $f_{c}$, as a function of particulate backscattering ratio using the Mobley et al. 2002 parameterization for Fournier-Forand scattering phase functions. Also shown is the fraction of scattered light collected by the LISST attenuation meter, $f_{c}$ LISST.

but at these signal levels it is possible to see the limits of the accuracy of the method $\left(\sim \pm 0.02 \mathrm{~m}^{-1}\right)$. For clear stations it would be hard to justify claiming significant improvement over standard (Flat and Zaneveld) correction procedures. Nonetheless, the relative success of the spectral matching for these sample spectra supports the idea that the optimized MC scattering correction procedure captures essential behavior of the instruments, including differences in imperfections between units.

One of the most novel aspects of the MC scattering correction approach is that it specifically deals with the scattering error for attenuation measurements. Figure 9a shows MC-corrected ac- 9 attenuation at $532 \mathrm{~nm}$ plotted against 532-nm LISST attenuation, also corrected using the MC approach. With a scattering collection angle of $\sim 0.027^{\circ}$ in water, the LISST measurement is as close to the true attenuation coefficient as we are currently able to achieve with commercially available instrumentation. Application of the MC correction to LISST data causes a small but potentially significant increase in attenuation values. Unfortunately, only a very small number of matchups are available at this time, greatly reducing the statistical significance of our analysis. However, if we are permitted to take these measurements at face value, some tentative conclusions can be reached. First of all, there is reasonable agreement between MC-corrected ac-9 attenuation and LISST attenuation, with the best-fit regression forced through the origin, indicating that MC-corrected ac- 9 attenuation is $\sim 4 \%$ higher than the MC-corrected LISST data. Uncorrected ac-9 attenuation was $\sim 20 \%$ lower than LISST data (best-fit regression slope $=0.8$ ). The coefficient of determination between MC-corrected ac-9 attenuation and LISST data $\left(r^{2}=0.86\right)$ is unchanged from that observed with uncorrected ac-9 attenuation, indicating that the observed spread in data is not induced by the application of the MC correction procedure. The limited number of data points available prevents a more detailed statistical analysis of these relationships. As a result we simply limit our conclusion to noting that there is reasonable agreement between MC-corrected ac-9 and MC-corrected LISST attenuation data.

Figure 9b shows standard uncorrected and MCcorrected ac-9 attenuation spectra and highlights the significant impact of scattering correction on attenuation signals (between 1.3 and 2.4 times higher). MC scattering correction has limited impact on the spectral shape of the attenuation signal, though there are subtle differences at 440 and $676 \mathrm{~nm}$, which correspond to phytoplankton absorption peaks. Figure $9 \mathrm{~b}$ also shows various estimates of the particulate scattering coefficient

TABLE 1. Best-fit polynomial coefficients for $f_{a}$ vs $b_{\mathrm{bp}} / b_{p}$ as a function of $r_{w}$ (Fig. 5). $f_{a}=a_{0}+a_{1} \cdot X+a_{2} \cdot X^{2}+a_{3} \cdot X^{3}+a_{4} \cdot X^{4}+a_{5} \cdot X^{5}+$ $a_{6} \cdot X^{6}$, where $X=b_{\mathrm{bp}} / b_{p}$.

\begin{tabular}{|c|c|c|c|c|c|c|c|}
\hline$r_{w}$ & $a_{0}$ & $a_{1}$ & $a_{2}$ & $a_{3}$ & $a_{4}$ & $a_{5}$ & $a_{6}$ \\
\hline 1 & $1.3298 \times 10^{-3}$ & $5.0680 \times 10^{0}$ & $-7.2323 \times 10^{1}$ & $1.4712 \times 10^{3}$ & $-1.9296 \times 10^{4}$ & $1.3474 \times 10^{5}$ & $-3.8071 \times 10^{5}$ \\
\hline 0.999 & $1.3731 \times 10^{-3}$ & $5.1371 \times 10^{0}$ & $-7.4330 \times 10^{1}$ & $1.5159 \times 10^{3}$ & $-1.9896 \times 10^{4}$ & $1.3898 \times 10^{5}$ & $-3.9246 \times 10^{5}$ \\
\hline 0.998 & $1.5710 \times 10^{-3}$ & $5.3242 \times 10^{0}$ & $-8.0867 \times 10^{1}$ & $1.6708 \times 10^{3}$ & $-2.2029 \times 10^{4}$ & $1.5423 \times 10^{5}$ & $-4.3644 \times 10^{5}$ \\
\hline 0.995 & $2.4926 \times 10^{-3}$ & $5.9851 \times 10^{0}$ & $-1.0596 \times 10^{2}$ & $2.2843 \times 10^{3}$ & $-3.0596 \times 10^{4}$ & $2.1593 \times 10^{5}$ & $-6.1399 \times 10^{5}$ \\
\hline 0.990 & $4.3554 \times 10^{-3}$ & $7.1043 \times 10^{0}$ & $-1.5041 \times 10^{2}$ & $3.3906 \times 10^{3}$ & $-4.6168 \times 10^{4}$ & $3.2858 \times 10^{5}$ & $-9.3905 \times 10^{5}$ \\
\hline 0.985 & $6.1369 \times 10^{-3}$ & $8.1460 \times 10^{0}$ & $-1.9228 \times 10^{2}$ & $4.4363 \times 10^{3}$ & $-6.0905 \times 10^{4}$ & $4.3527 \times 10^{5}$ & $-1.2470 \times 10^{6}$ \\
\hline 0.980 & $7.9184 \times 10^{-3}$ & $9.1877 \times 10^{0}$ & $-2.3415 \times 10^{2}$ & $5.4819 \times 10^{3}$ & $-7.5643 \times 10^{4}$ & $5.4196 \times 10^{5}$ & $-1.5550 \times 10^{6}$ \\
\hline 0.970 & $1.1263 \times 10^{-2}$ & $1.1073 \times 10^{1}$ & $-3.1117 \times 10^{2}$ & $7.4140 \times 10^{3}$ & $-1.0292 \times 10^{5}$ & $7.3961 \times 10^{5}$ & $-2.1259 \times 10^{6}$ \\
\hline 0.960 & $1.4403 \times 10^{-2}$ & $1.2790 \times 10^{1}$ & $-3.8228 \times 10^{2}$ & $9.2046 \times 10^{3}$ & $-1.2824 \times 10^{5}$ & $9.2320 \times 10^{5}$ & $-2.6564 \times 10^{6}$ \\
\hline 0.950 & $1.7371 \times 10^{-2}$ & $1.4361 \times 10^{1}$ & $-4.4831 \times 10^{2}$ & $1.0874 \times 10^{4}$ & $-1.5188 \times 10^{5}$ & $1.0948 \times 10^{6}$ & $-3.1523 \times 10^{6}$ \\
\hline
\end{tabular}



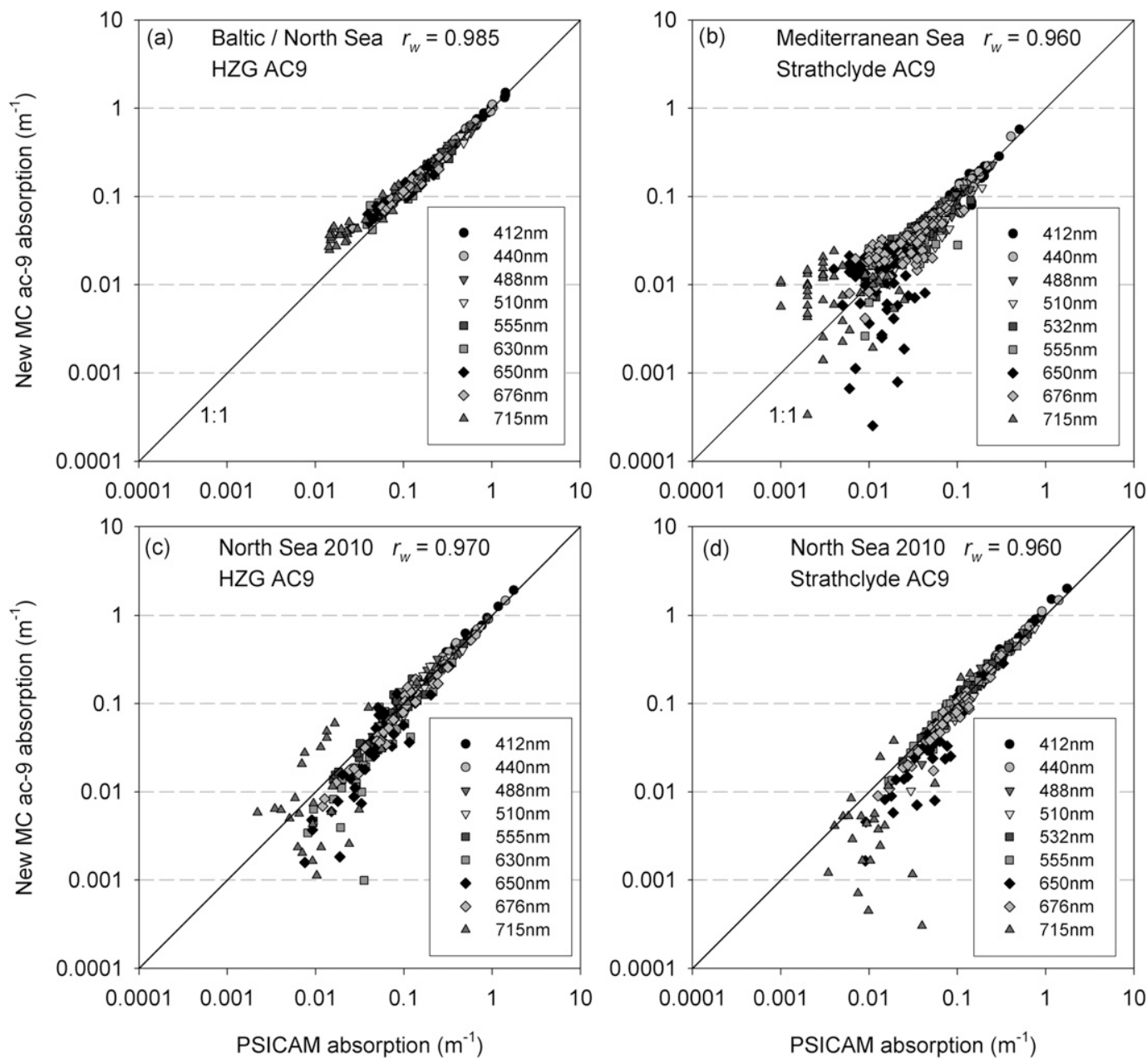

FIG. 7. New ac-9 absorption data obtained by selection of optimal values of $r_{w}$ for each dataset do not suffer from systematic overestimation and provide significantly better matches with PSICAM absorption values.

using combinations of corrected and uncorrected attenuation and various absorption estimates. The most notable feature is that the increase in attenuation coefficient roughly translates into a similar magnitude increase in particulate scattering. The particulate scattering spectral shape is broadly similar for all correction methods, but there is some sign of enhanced depressions at 440 and $676 \mathrm{~nm}$, where the effects of anomalous dispersion associated with phytoplankton absorption peaks could be significant (van de Hulst 1957). We note that this result contradicts McKee et al. (2008), who found that the increase in attenuation was largely associated with corresponding increases in absorption. The effect of incorporating wall effects into the Monte Carlo analysis has been to reduce the magnitude of retrieved absorption signals while attenuation signals remain high. Consequently, since $b_{p}(\lambda)=c_{n}(\lambda)-a_{n}(\lambda)$, there is a corresponding increase in estimates of $b_{p}(\lambda)$, in this case by a factor of $\sim 2$.

\section{Discussion}

The availability of validated absorption data from the PSICAM has enabled a first assessment of the performance of ac- 9 absorption measurements and associated scattering correction methods using field data. Results presented in Figs. 3 and 8 demonstrate serious deficiencies in standard scattering correction methods (Flat and Zaneveld) as well as the original iterative Monte Carlo scattering correction (McKee et al. 2008). The observed errors in ac- 9 absorption after standard scattering corrections are broadly in line with those described by Leymarie et al. (2010) in terms of spectral shape (e.g., their Fig. 7g). We routinely observe nonzero absorption signals at $715 \mathrm{~nm}$, consistent with earlier studies (Doxaran et al. 2007; Röttgers et al. 2008), which contravenes one of the fundamental assumptions of the lat and Zaneveld correction methods. The second assumption for these correction methods, that the scattering 

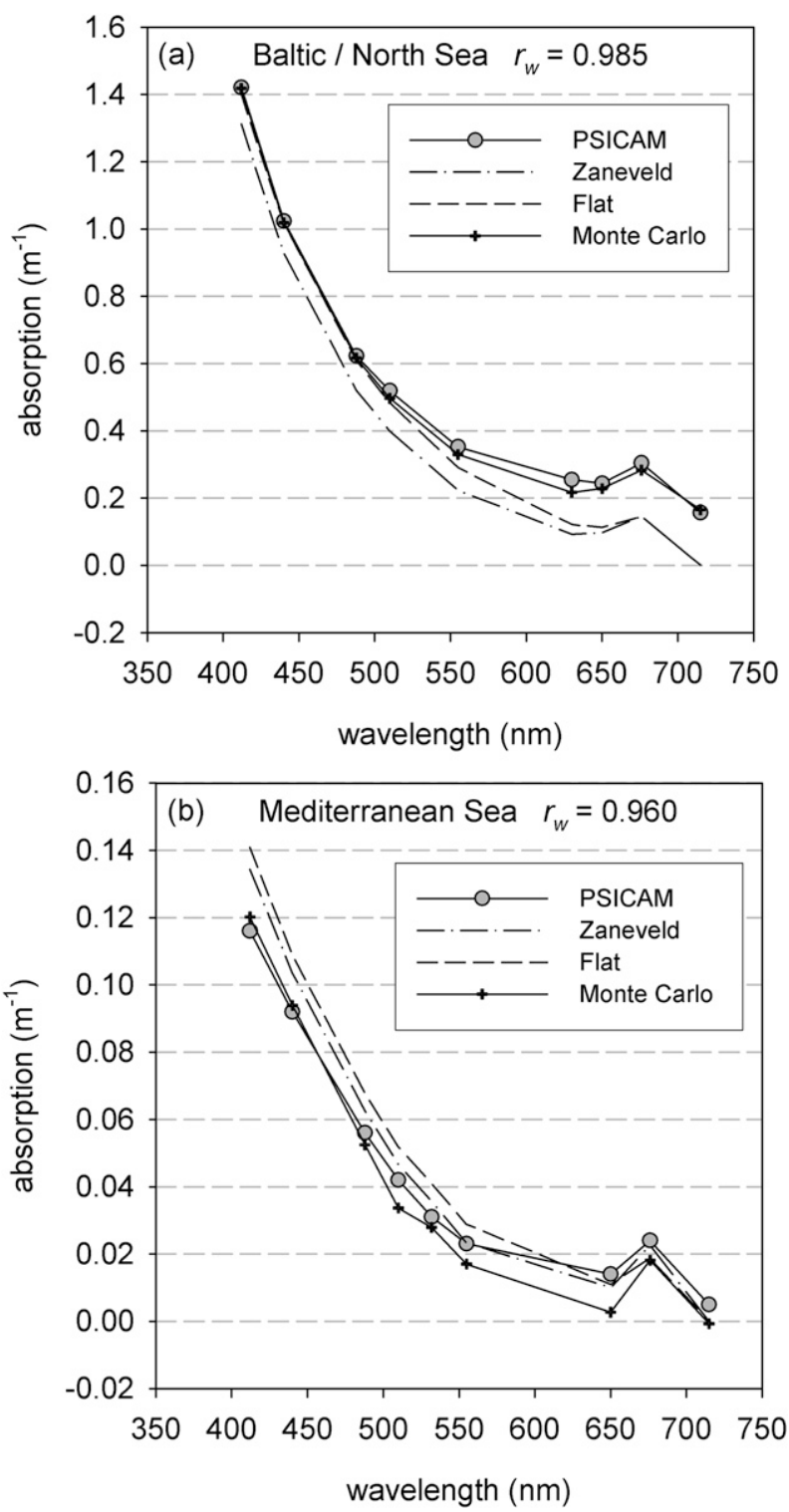

FIG. 8. PSICAM and ac-9 absorption spectra for one station from (a) the Baltic/North Sea and (b) the Mediterranean Sea. Data from ac- 9 are presented before and after application of a number of proposed scattering correction procedures. Note order of magnitude difference between $y$ axes in plots (a) and (b).

phase function is wavelength independent, is also in doubt since some studies in coastal waters have observed significant wavelength dependence in either measured volume scattering functions or in particulate backscattering ratios (Chami et al. 2006; McKee et al. 2009). Errors for ac- 9 absorption data processed using the Flat or Zaneveld corrections will be significant in turbid coastal waters, where NIR nonwater absorption is greatest and there is significant particulate scattering. Although NIR nonwater absorption levels are much lower in oceanic waters than coastal waters, assuming zero NIR nonwater absorption and using this for baseline correction may introduce a significant error in the estimation of nonwater absorption at visible wavelengths. Our ability to resolve these nonzero NIR absorption signals with the MCcorrected ac-9 unfortunately remains limited for clear oceanic waters because of unresolved measurement errors at low signal levels. Percentage error becomes significant for absorption signals below $\sim 0.02 \mathrm{~m}^{-1}$ (Fig. 7), probably owing to limits in the signal to noise of the instruments. Despite this, we would recommend use of the improved MC correction method for all new datasets, including clear oceanic datasets, for the potentially significant impact on attenuation signals and for the sake of data consistency.

The Monte Carlo ac-9 scattering correction method will be sensitive to errors in the estimation of $b_{\mathrm{bp}}(\lambda) /$ $b_{p}(\lambda)$ (Chami et al. 2006), which have been shown to increase substantially for low scattering signal levels (McKee et al. 2009). The improved MC correction method gives an average RMSE of $27 \%$ for all North Sea 2010 data points using standard $b_{\mathrm{bp}}(\lambda)$ measurements. Sensitivity to potential errors in backscattering measurements used in the MC correction method was tested by artificially increasing $b_{\mathrm{bp}}(\lambda)$ values by $10 \%$, $20 \%$, and $50 \%$, resulting in a new average RMSE of $33 \%$, $40 \%$, and $65 \%$, respectively. We note that Boss et al. 2004 suggest $10 \%$ error limits for backscattering data corrected using standard approaches. From this we can conclude that the revised MC correction method is not overly sensitive to errors in $b_{\mathrm{bp}}(\lambda)$ measurements for the range of values encountered in these datasets. This is consistent with consideration of the curves shown in Fig. 6, which suggest that the greatest sensitivity will occur for very low values of $b_{\mathrm{bp}}(\lambda) / b_{p}(\lambda)$. There remains the potential impact of artifacts in pure water calibration and instrument drift between calibrations. Pure water blank errors are likely to be more significant when measured absorption signals are low and could become the limiting factor in ac- 9 accuracy once scattering errors are properly accounted for.

We have used the Mobley et al. (2002) parameterization for Fournier-Forand scattering phase functions throughout this work for the convenience of being able to relate the VSF to $b_{\mathrm{bp}} / b_{p}$. However, this parameterization assumes a linear relationship between the mean refractive index of the particle population, $n_{r}$, and the slope of the particle size distribution ( $\mu$, assuming a Junge distribution) that was also selected for convenience rather than necessarily reflecting an observation of nature. There is therefore a need to consider the potential impact of alternative VSF parameterizations on the MC ac-9 correction method. Fournier-Forand 

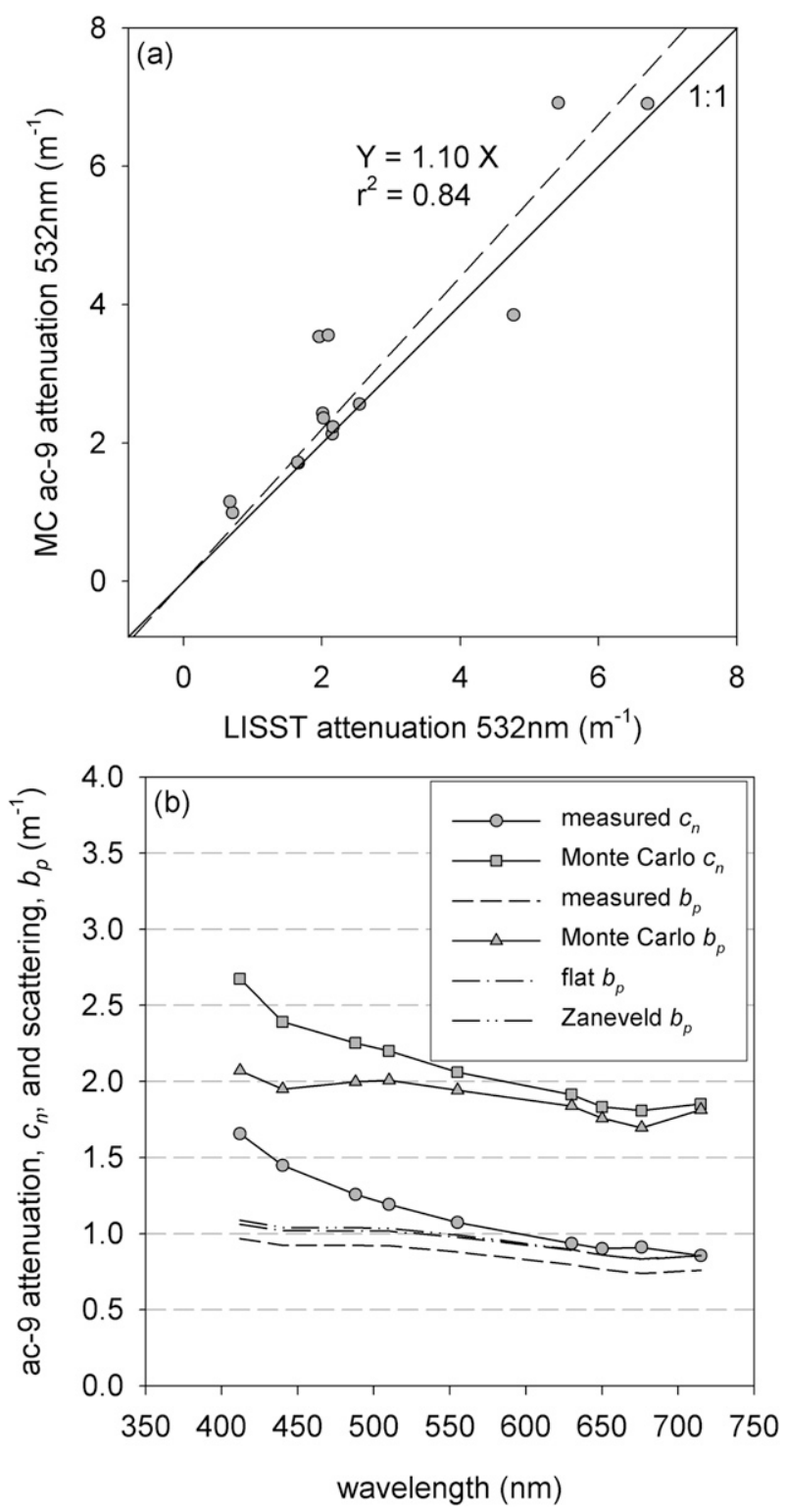

FIG. 9. (a) MC-corrected ac-9 and LISST attenuation at $532 \mathrm{~nm}$ show reasonably close agreement. (b) MC-corrected attenuation is significantly higher than the standard ac- 9 measurement across the visible spectrum, and results in higher values of scattering coefficient with enhanced spectral features associated with anomalous dispersion.

scattering phase functions were calculated for a wide range of combinations of $\mu(3.01-5)$ and $n_{r}(1.01-1.20)$ and corresponding values of $f_{a}$ and $f_{c}$ obtained for the ac-9 from Eqs. (8) and (9). The absorption function $f_{a}$ was obtained assuming $r_{w}=1$, and Fig. 10 shows results for all combinations of $n_{r}$ and $\mu$ that return phase functions with $b_{\mathrm{bp}} / b_{p}<0.1$. The only significant deviation in $f_{a}$ from the results obtained with the Mobley et al. parameterization occurs when refractive index is very low
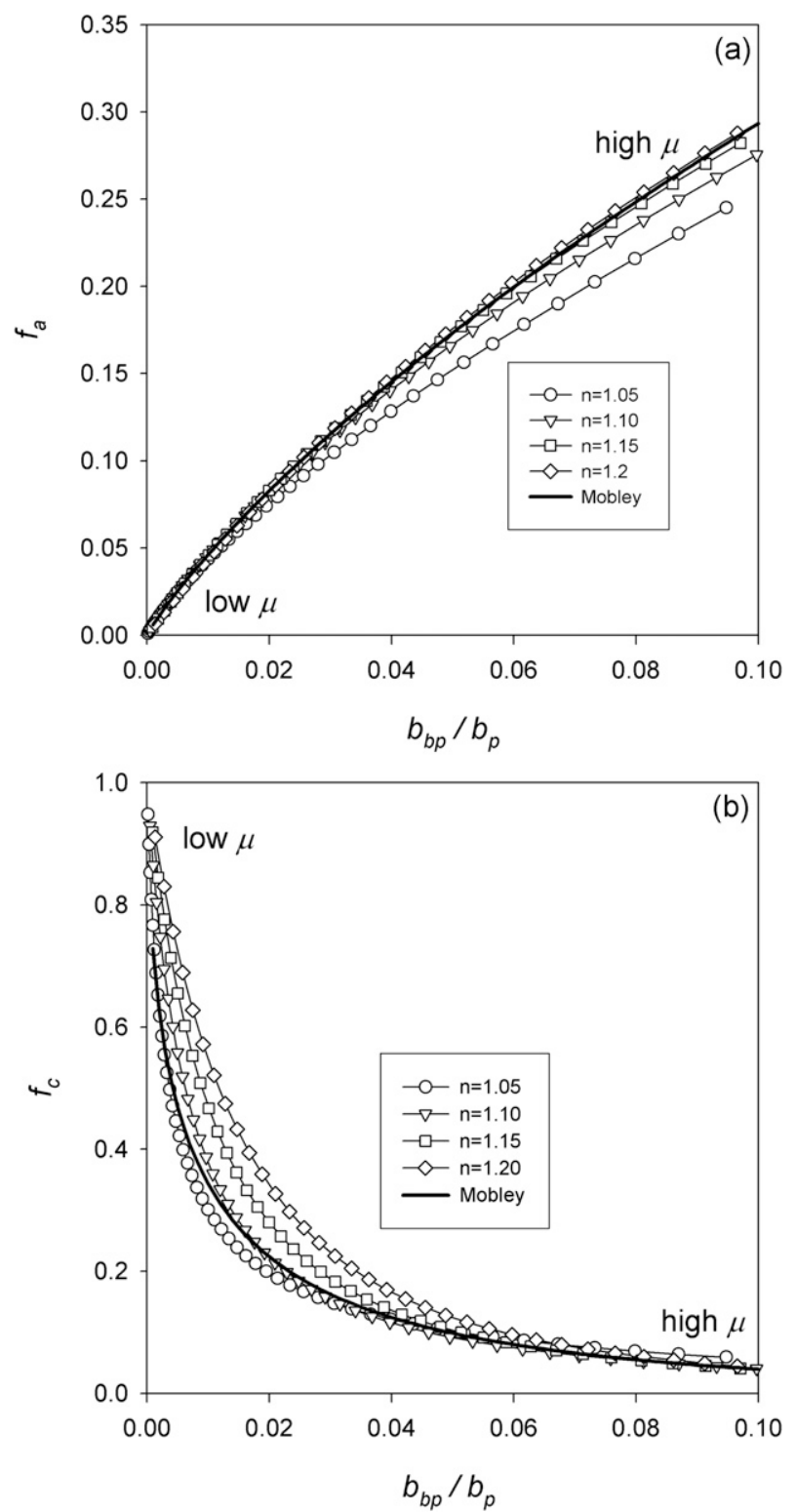

FIG. 10. (a) Alternative parameterizations of the FournierForand phase function have little impact on determination of $f_{a}$ except when refractive index is low and the backscattering ratio is greater than 0.03. (b) Attenuation sensor has greater sensitivity to the parameterization of the Fournier-Forand phase function.

and $b_{\mathrm{bp}} / b_{p}$ is very high, a most unusual situation in nature (Fig. 10a). The attenuation error $f_{c}$, on the other hand, shows considerably greater sensitivity to the choice of phase function parameterization (Fig. 10b). Since both $f_{a}$ and $f_{c}$ are used to correct absorption and attenuation data [Eqs. (6) and (7)], there is a reasonable concern that the choice of phase function parameterization might influence the performance of the MC ac-9 correction method. It is unlikely, however, that this would be sufficient to account for the effects that have 
been attributed by us to wall reflectance artifacts, and the experimental results in Fig. 4 remain convincing evidence in support of our hypothesis. Nevertheless, there may be scope in the future to include the effect of alternative phase function parameterization to form part of an associated uncertainty analysis estimate for the MC ac-9 method.

The effect of scattering collection error on ac- 9 attenuation measurements has been shown to be considerable, with uncorrected ac- 9 attenuation systematically underestimating the true value by a factor of $\sim 0.8$ (from best-fit regression). This value is close to the top end of the range presented by Boss et al. (2009), who observed underestimates of $50 \%-80 \%$ between ac- 9 and LISST attenuation data. Equation (9) and Fig. 6 demonstrate the sensitivity of the attenuation scattering collection error to variations in the magnitude of the scattering phase function, so it is quite feasible that this could account for some of the range found by Boss et al. (2009). Leymarie et al. (2010) have also pointed out that the percentage error in attenuation measurements is also dependent on the relative abundance of scattering to absorption. In this work we are primarily interested in attenuation by dissolved materials and suspended particles. Bogucki et al. (1998) demonstrated the influence of turbulence on scattering at very small forward angles. It is possible that some of the observed spread between LISST and ac-9 attenuation data could be associated with different sensitivity to the effects of turbulence.

The original Monte Carlo-based scattering correction procedure (McKee et al. 2008) has also been found to be deficient, generating systematic overestimates of absorption at all wavelengths (Fig. 3). Significantly improved results were obtained by including in the Monte Carlo simulation scheme for the reflecting-tube meter an additional loss term representing imperfect reflectance at the glass wall of the flow tube. However, its introduction imposes new restrictions on the applicability of the Monte Carlo scattering correction method and raises questions about the consistency of data between ac-9 sensors. Assuming that these results do indeed indicate significant differences in optical performance between ac-9 units, it is reasonable to wonder how such differences might come about and what influence they might have for future correction strategies. Knowing the exact nature of the imperfections may result in improvement of the Monte Carlo model of the flow tube and therefore even better error correction. Imperfections in the glass walls of the reflecting flow tube are potentially inherent in the manufacturing process and could result from polishing and finishing of the glass itself as well as effects of instrument construction. Degradation of the exterior surface of the glass flow tube where total internal reflectance occurs and impact of mechanical fixing points on the reflectance properties of the glass flow tube remain uncertain factors. A major concern is that there could be deterioration in the optical quality of the glass flow tube over time because of the effects of exposure to the marine environment and cleaning operations. Given the predicted sensitivity to wall reflectance imperfections, great care should be taken to minimize impact on the glass walls of the absorption tube. This raises the real possibility that the calibration of the instrument will change over time (beyond, e.g., changes in lamp output), limiting the potential for backward application of this methodology to historic datasets.

Taken in the round, all of these results reemphasize the need for regular calibration and validation of ac-9 data. This has to go beyond calibration with ultrapure water to establish pure water offsets and must include assessment of the quality of absorption data measured in the presence of particle scattering. The method presented in this paper involves comparison of measured ac-9 data with samples measured with a benchtop PSICAM. Although integrating-cavity absorption instruments like this are becoming more common, many laboratories do not possess the technology and many will prefer to wait until in situ absorption cavity absorption meters have been robustly demonstrated to work or will have to wait until funds become available. For groups such as these, there is an urgent need for an alternative calibration/validation procedure. One possibility would be to perform a set of calibrations with a pigment solution of known absorption coefficient (measured on a benchtop spectrophotometer) and to measure the effect of adding a pure scattering material, for example, barium sulfate. A similar procedure was used to validate the HZG PSICAM (Röttgers et al. 2005) and could perhaps be evolved to facilitate estimation of $r_{w}$ for the modified ac-9 Monte Carlo procedure proposed here. Caution is required in the choice of materials used in such a process, as some of the ac-9 components may be damaged by solvents or dyes. Further work is required in this respect.

Quantitative IOP measurements are essential for radiative transfer modeling and interpretation of ocean color remote sensing signals. A new generation of integrating-cavity absorption meters (Röttgers et al. 2005; Pope and Fry 1997) is transforming the accuracy and sensitivity of oceanographic absorption measurements. Similar improvements are urgently required for multior hyperspectral attenuation measurements. The LISST is a single-wavelength device with a short $(5 \mathrm{~cm})$ pathlength and is still subject to scattering collection errors (e.g., Figure 6), albeit at much lower levels than the ac-9. 
Given the magnitude of the observed errors for both attenuation and particulate scattering, development of a new generation of spectral attenuation meters that minimize or eliminate scattering collection error should be a priority.

\section{Conclusions}

An updated version of the iterative Monte Carlo scattering correction method for ac-9 absorption and attenuation data has been developed and shown to provide significantly better quality data than standard scattering correction methods. The requirement for concurrent backscattering measurements adds an additional cost and processing burden compared to standard correction methods, though we note the use of backscattering sensors is becoming increasingly common. Its general applicability is currently further limited to those situations in which an independent, robust absorption measurement on water samples is available. This situation could be improved through development of a robust benchtop spectrophotometric calibration/validation method. The introduction of a new generation of benchtop integrating-cavity absorption meters is naturally being followed by submersible versions (e.g., HOBI Labs $a$-Sphere and TriOS OSCAR flow through PSICAMs). In situ versions of these instruments need to be thoroughly tested and validated both optically and in terms of practical considerations for in-water deployments, for example, biofouling properties and flush times. It is impossible to predict if or how long it will take for this type of technology to replace the ac-9/ac-s generation, but it seems likely that there will be a need for the type of correction presented in this paper for some years to come. Very importantly, there is also a large volume of historic ac-9 data that needs to be reassessed in light of these results and the predictions of other modeling studies (Leymarie et al. 2010). There is an urgent need for improved scattering correction methods that can address some of the fundamental weaknesses of standard scattering correction methods, but that do not require the full range of additional data (e.g., backscattering and PSICAM data) needed for the updated Monte Carlo scattering correction presented here. The extent to which such a correction procedure can be established for historic (and potentially current) datasets will form the basis for future work in this area.

Acknowledgments. The authors acknowledge support from the following funding bodies: NERC Advanced Fellowship NE/E013678/1 (McKee), several awards from the Royal Society of Edinburgh International Exchange Programme (Piskozub and McKee), the European Space
Agency under the "Water Radiance" Project ESA/ ESTEC 22192/09/NL/CT (Röttgers and McKee), and the Office of Naval Research Optics and Biology Award N00014-05-1-0246 to D. Stramski (Reynolds). Dr. Chuck Trees from the NATO Underwater Research Centre, La Spezia, kindly provided access to facilities on board R/V Alliance for the BP09 cruise in the Mediterranean Sea. Dr. Sławomir Woźniak generously provided access to facilities on board R/V Oceania. We thank Vanessa Wright for her assistance with measurements on board the Oceania.

\section{REFERENCES}

Bogucki, D. J., J. A. Domaradzki, D. Stramski, and J. R. V. Zaneveld, 1998: Comparison of near-forward light scattering on oceanic turbulence and particles. Appl. Opt., 37, 46694677.

Boss, E., and W. S. Pegau, 2001: Relationship of light scattering at an angle in the backward direction to the backscattering coefficient. Appl. Opt., 40, 5503-5507.

,,-- M. Lee, M. Twardowski, E. Shybanov, G. Korotaev, and F. Baratange, 2004: Particulate backscattering ratio at LEO 15 and its use to study particle composition and distribution. J. Geophys. Res., 109, C01014, doi:10.1029/2002JC001514.

—, W. H. Slade, M. Behrenfeld, and G. Dall'Olmo, 2009: Acceptance angle effects on the beam attenuation in the ocean. Opt. Express, 17, 1535-1550.

Buiteveld, H., J. H. M. Hakvoort, and M. Donze, 1994: The optical properties of pure water. Ocean Optics XII, J. S. Jaffe, Ed., International Society for Optical Engineering (SPIE Proceedings, Vol. 2258), 174-183.

Chami, M., E. B. Shybanov, G. A. Khomenko, M. E.-G. Lee, O. V. Martynov, and G. K. Korotaev, 2006: Spectral variation of the volume scattering function measured over the full range of scattering angles in a coastal environment. Appl. Opt., 45, 3605-3619.

Doxaran, D., M. Babin, and E. Leymarie, 2007: Near-infrared light scattering by particles in coastal waters. Opt. Express, 15, 12 834-12849.

Flatau, P. J., J. Piskozub, and J. R. Zaneveld, 1999: Asymptotic light field in the presence of a bubble-layer. Opt. Express, 5, $120-123$.

Freda, W., and J. Piskozub, 2007: Improved method of FournierForand marine phase function parameterization. Opt. Express, 15, $12763-12768$.

Hakvoort, J. H. M., and R. R. Wouts, 1994: Monte Carlo modeling of the light field in reflective-tube-type absorption meters. Ocean Optics XII, J. S. Jaffe, Ed., International Society for Optical Engineering (SPIE Proceedings, Vol. 2258), 529538.

Jonasz, M., and G. Fournier, 1996: Approximation of the size distribution of marine particles by a sum of log-normal functions. Limnol. Oceanogr., 41, 744-754.

Kirk, J. T. O., 1992: Monte Carlo modeling of the performance of a reflective tube absorption meter. Appl. Opt., 31, 6463 6468.

Leymarie, E., D. Doxaran, and M. Babin, 2010: Uncertainties associated to measurements of inherent optical properties in natural waters. Appl. Opt., 49, 5415-5436. 
Maffione, R. A., and D. R. Dana, 1997: Instruments and methods for measuring the backward-scattering coefficient of ocean waters. Appl. Opt., 36, 6057-6067.

McKee, D., and A. Cunningham, 2005: Evidence for wavelength dependence of the scattering phase function and its implication for modeling radiance transfer in shelf seas. Appl. Opt., 44, 126-135.

$\longrightarrow, \ldots$, and S. Craig, 2003: Semi-empirical correction algorithm for AC-9 measurements in a coccolithophore bloom. Appl. Opt., 42, 4369-4374.

_ , J. Piskozub, and I. Brown, 2008: Scattering error corrections for in situ absorption and attenuation measurements. Opt. Express, 16, 19480-19492.

—, M. Chami, I. Brown, V. S. Calzado, D. Doxaran, and A. Cunningham, 2009: Role of measurement uncertainties in observed variability in the spectral backscattering ratio: A case study in mineral-rich coastal waters. Appl. Opt., 48, 46634675.

Mobley, C. D., 1994: Light and Water: Radiative Transfer in Natural Waters. Academic Press, 592 pp.

— , L. K. Sundman, and E. Boss, 2002: Phase function effects on oceanic light fields. Appl. Opt., 41, 1035-1050.

Morel, A., 1974: Optical properties of pure water and pure seawater. Optical Aspects of Oceanography, N. G. Jerlov and E. S. Nielsen, Eds., Academic, 1-24.

Pegau, W. S., D. Gray, and J. R. V. Zaneveld, 1997: Absorption and attenuation of visible and near-infrared light in water: Dependence on temperature and salinity. Appl. Opt., 36, 6035-6046.

Piskozub, J., and D. McKee, 2011: Effective scattering phase functions for the multiple scattering regime. Opt. Express, 19, 4786-4794.

_, P. J. Flatau, and J. R. V. Zaneveld, 2001: Monte Carlo study of the scattering error of a quartz reflective absorption tube. J. Atmos. Oceanic Technol., 18, 438-445.

—, D. Stramski, E. Terrill, and W. K. Melville, 2004: Influence of forward and multiple light scatter on the measurement of beam attenuation in highly scattering marine environments. Appl. Opt., 43, 4723-4731.

— sensing: Choosing the correct depth weighting function. Opt. Express, 16, 14683-14688.

Pope, R. M., and E. S. Fry, 1997: Absorption spectrum (380$700 \mathrm{~nm}$ ) of pure water. II. Integrating cavity measurements. Appl. Opt., 36, 8710-8723.

Reynolds, R. A., D. Stramski, V. M. Wright, and S. B. Woźniak, 2010: Measurements and characterization of particle size distributions in coastal waters. J. Geophys. Res., 115, C08024, doi:10.1029/2009JC005930.

Risović, D., 2002: Effect of suspended particulate-size distribution on the backscattering ratio in the remote sensing of seawater. Appl. Opt., 41, 7092-7101.

Röttgers, R., and R. Doerffer, 2007: Measurements of optical absorption by chromophoric dissolved organic matter using a point-source integrating-cavity absorption meter. Limnol. Oceanogr. Methods, 5, 126-135.

—, W. Schönfeld, P.-R. Kipp, and R. Doerffer, 2005: Practical test of a point-source integrating cavity absorption meter: The performance of different collector assemblies. Appl. Opt., 44, 5549-5560.

, C. Häse, and R. Doerffer, 2007: Determination of the particulate absorption of microalgae using a point-source integrating-cavity absorption meter: Verification with a photometric technique, improvements for pigment bleaching and correction for chlorophyll fluorescence. Limnol. Oceanogr. Methods, 5, 1-12.

—, A. Bracher, S. Gehnke, B. Schmitt, and S. Wozniak, 2008: Light absorption by natural aquatic particles in the nearinfrared (700-900 nm) spectral region. Ocean Optics XIX Conf., Barga, Italy, Oceanography Society, 540.

Smith, R. C., and K. S. Baker, 1981: Optical properties of the clearest natural waters (200-800 nm). Appl. Opt., 20, 177-184.

Stramski, D., and J. Piskozub, 2003: Estimation of scattering error in spectrophotometric measurements of light absorption by aquatic particles from three-dimensional radiative transfer simulations. Appl. Opt., 42, 3634-3646.

— concentration of particulate organic carbon and optical properties in the eastern South Pacific and eastern Atlantic Oceans. Biogeosciences, 5, 171-201.

Twardowski, M. S., H. Claustre, S. A. Freeman, D. Stramski, and Y. Huot, 2007: Optical backscattering properties of the "clearest" natural waters. Biogeosciences, 4, 1041-1058.

van de Hulst, H. C., 1957: Light Scattering by Small Particles. John Wiley and Sons, $470 \mathrm{pp}$.

Voss, K. J., and R. W. Austin, 1993: Beam-attenuation measurements error due to small-angle scattering acceptance. J. Atmos. Oceanic Technol., 10,113-121.

Zaneveld, J. R. V., J. C. Kitchen, and C. M. Moore, 1994: The scattering error correction of reflecting-tube absorption meters. Ocean Optics XII, J. S. Jaffe, Ed., International Society for Optical Engineering (SPIE Proceedings, Vol. 2258), 44-55. 
Copyright of Journal of Atmospheric \& Oceanic Technology is the property of American Meteorological Society and its content may not be copied or emailed to multiple sites or posted to a listserv without the copyright holder's express written permission. However, users may print, download, or email articles for individual use. 